Radial and Nonradial Pulsations as Probes of Stellar Physics

ASP Conference Series, Vol. 259, 2002

C. Aerts, T.R. Bedding, 8 J. Christensen-Dalsgaard, eds.

\title{
$\delta$ Scuti Variables in the HR Diagram
}

\author{
E. Rodríguez
}

Instituto de Astrofísica de Andalucía, CSIC, P.O. Box 3004, E-18080 Granada, Spain

\section{Breger}

Institut für Astronomie, Universität Wien, Türkenschanzstr. 17, A-1180, Austria

The location of the $\delta$ Scuti variables in the HR diagram is discussed on the basis of HIPPARCOS parallaxes and $u v b y \beta$ photometry together with the R00 catalogue (Rodríguez et al., 2000). In particular, the properties of the $\delta$ Scutitype pulsators with nonsolar surface abundances (SX Phe, $\lambda$ Boo, $\rho$ Pup, $\delta$ Del and classical Am stars subgroups) are examined.

The latter four classes of stars are spectroscopically defined subclasses with surface abundance anomalies. Although the stars $\rho$ Pup and $\delta$ Del are also pulsators, the groups named after these stars should not be regarded as pulsation subclasses of $\delta$ Scuti stars. The abundance anomalies in these stars affect the pulsation properties, e.g., the classical Am stars are constant in light or show only small pulsation amplitudes (e.g., HD 1097). These four groups are only a small selection from the bewildering zoo of stars with unusual surface abundances in this temperature region and represent the groups with $\delta$ Scuti pulsation.

$\lambda$ Boo stars are metal-poor Pop. I objects with spectral types from late-B to mid-F. These stars cover the whole main-sequence range between the zero-age and the terminal-age main sequence. Both the $\lambda$ Boo stars and the SX Phe variables show metal-poor spectra and positive $\delta \mathrm{m}_{1}$ indices in the $u v b y$ system. This can make it difficult to classify field stars correctly if detailed spectroscopic or space velocity information is not available. The status of BS Tuc (HD 6870) is now clear: it is not an SX Phe variable but a strong $\lambda$ Boo star (hF0A1V). Good agreement between the photometric and HIPPARCOS absolute magnitudes is found for the $\lambda$ Boo stars. Thus, the photometric $u v b y \beta$ calibrations are applicable for these variables, but we find that they should not be used for the group of evolved metallic-line A stars.

The SX Phe variables are the $\delta$ Scuti stars of Pop. II and old disk population. Since such old stars at $\sim 8500 \mathrm{~K}$ should have already evolved away and no longer exist in this part of the HR diagram, they are also unusual from an evolutionary point of view. The absolute magnitudes were calculated from the calibrations of $u v b y \beta$ photometry as well as HIPPARCOS parallaxes. However, only one SX Phe field star (SX Phe itself) has an accurate HIPPARCOS parallax. For SX Phe, the photometric and HIPPARCOS absolute magnitudes differ by $0 .^{m} 8$ : this may be caused by systematic errors in applying the Pop. I calibrations to 
this extremely metal-poor star. Such an uncertainty in the photometric absolute magnitudes, though of smaller size, may also apply to the other stars of this group.

When the HIPPARCOS and photometric absolute magnitudes are compared, the agreement is not satisfactory: the HIPPARCOS parallaxes indicate, on the average, higher luminosities of $\sim 0{ }^{m} 4$. The deviations arise in stars with low rotational velocities and/or high metallicities (low $\delta \mathrm{m}_{1}$ indices). The metallic-line stars are usually slow rotators, so that it is not easy to separate the two effects. Since the HIPPARCOS parallaxes should not be affected by these stellar properties, the photometric luminosity calibrations must be in error for these stars. However, good agreement is found for fast rotators and it is also shown that systematic deviations exist for the photometrically determined absolute magnitudes, which correlate with $v \sin i$ and $\delta \mathrm{m}_{1}$. The situation may be summarized as follows: for $\delta$ Scuti stars with normal metals and rotational velocities of $100 \mathrm{~km} \mathrm{~s}^{-1}$, the photometric calibrations are in agreement with the HIPPARCOS parallaxes. For the other stars, systematic corrections need to be applied to the photometrically derived values of $\mathrm{M}_{v}$, but the size of these corrections still needs to be determined.

Finally, the $\delta$ Scuti-type variables are located in the HR diagram using the information discussed before and new borders of the classical instability strip for the $\delta$ Scuti region are determined: the observational red edge used in the R94 catalogue (Rodríguez et al., 1994) seems to be well defined (this is the same edge used by Breger, 1979), whereas some stars suggest that the observational blue edge, used in the R94 catalogue, must be shifted bluewards by about $100 \mathrm{~K}$.

\section{References}

Breger, M. 1979, PASP, 91, 5

Rodríguez, E., López de Coca, P., Rolland, A., Garrido, R., \& Costa, V. 1994, A\&AS, 106, 21

Rodríguez, E., López-González, M. J., \& López de Coca, P. 2000, A\&AS, 144, 469 